\title{
When Two Is Better than One: Differences in Characteristics of Women Using Condoms Only Compared to Those Using Condoms Combined with an Effective Contraceptive
}

\author{
Jason Ong, MMed, ${ }^{1,5}$ Meredith Temple-Smith, $\mathrm{PhD},{ }^{2}$ William Wong, MD, ${ }^{3}$ \\ Kathleen McNamee, $\mathrm{MPH}^{4}$ and Christopher Fairley, $\mathrm{PhD}^{1,5}$
}

\begin{abstract}
Background: There remain high rates of unintended pregnancy around the world. Adding an effective contraceptive to those who currently only use male condoms may reduce these rates. The aim of this study is to identify the prevalence of and factors associated with the combination use of an effective contraception with male condoms in sexually active women who are already using male condoms.

Methods: Women attending Family Planning Victoria Clinics from April to July 2011 were approached to complete a questionnaire about contraception usage in the last 3 months and 34 associated variables. Univariate and multivariate analyses were conducted to determine women with greater odds of an effective contraception together with male condoms compared with those using male condoms only.

Results: Of 1006 women surveyed, 872 women stated it was "very important" or "important" to avoid pregnancy at this stage of their life. Of these 872 women, 690 reported male condom use-274 women used male condoms and an effective contraception, while 416 used male condoms only. Of note, only $67(16 \%)$ of the 416 solely male condom users were using this consistently. On multivariate analysis, characteristics associated with combination use (compared with condom use only) were discussion with a health professional in the last 12 months (adjusted odds ratio [AOR] 2.9; 95\% confidence interval [CI] 1.9, 4.4), satisfaction with contraception (AOR 1.8; 95\% CI 1.3, 2.7), having more than 1 partner in the last 3 months (AOR 1.8; 95\% CI 1.2, 2.6) and past pregnancy (AOR 0.3; 95\% CI 0.1, 0.5).

Conclusions: In a group of women not intending to be pregnant who were using male condoms, a significant number remained at risk for unintended pregnancy due to inconsistent use of male condoms and poor use of concurrent effective contraception.
\end{abstract}

\section{Introduction}

$\mathbf{T}_{\mathrm{p}}$ HE RATES OF TEENAGE CONCEPTION and unintended pregnancy continue to be an issue in Australia. ${ }^{1,2}$ Use of male condoms alone provides protection from sexually transmissible infections (STIs) and pregnancy; however, the typical contraceptive failure rate within the first year of use is $18 \%{ }^{3}$ Combining a condom with another effective contraceptive method protects more effectively against unintended pregnancy than condoms alone. ${ }^{4}$ For pill takers, the concur- rent use of condoms may reduce the pill's failure rate by compensating for high rates of inconsistent pill use, ${ }^{5}$ with typical failure rates of $9 \%$ during the first year of use (compared with $0.3 \%$ with perfect use). ${ }^{6}$

Despite the benefits of using condoms and an effective contraception concurrently, it remains "one of the most under-rated and under-promoted public health practices today. ${ }^{\prime 7}$ In countries that likewise share a high rate of STIs and unintended pregnancy, ${ }^{8-14}$ low use of an effective contraception with male condoms are also consistently reported. In

\footnotetext{
${ }^{1}$ Melbourne School of Population and Global Health and ${ }^{2}$ General Practice and Primary Health Care Academic Centre, University of Melbourne, Melbourne, Australia.

${ }^{3}$ Department of Family Medicine and Primary Care, University of Hong Kong, Hong Kong.

${ }^{4}$ Family Planning Victoria. Monash Medical Centre, Department of Obstetrics and Gynaecology, Clayton, Australia.

${ }^{5}$ Melbourne Sexual Health Centre, Melbourne, Australia.
} 
Australia, fewer than one in eight contraceptive users reported this combination. ${ }^{15}$

The infrequent use of male condoms with an effective contraception may be due to a variety of reasons. There may be a false understanding of the efficacy of condoms in preventing pregnancy, ${ }^{16}$ the relative difficulty in accessing an effective contraception (requiring a visit to a health professional) compared with purchasing condoms (does not require a visit to a health professional), or simply a lack of knowledge of the availability of effective contraceptives. ${ }^{17}$ Multiple factors related to acceptability, side effects, adherence, and perceptions of the various effective contraceptives may also play a role. ${ }^{18}$ In explaining the low rate of combination use from the provider's viewpoint, there may be concerns about how realistic it is to motivate the use of two methods simultaneously, fear that adding a second method may impair consistency of use of the first, and that using two methods may not be financially or logistically feasible, either for the client or for public health programs. ${ }^{19}$

To understand why this practice is uncommon in Australia, we examined the differences between single versus dual contraceptive users among a group of women who stated they were not intending to conceive. This information may be useful in developing strategies to encourage the combined use of an effective contraceptive and male condoms in a group of women at high risk for both STIs and unintended pregnancy.

The data was obtained from a larger study examining contraceptive use in women attending a family planning clinic. An unexpectedly large proportion of women reported use of dual methods, providing an opportunity to report these findings.

\section{Methods}

\section{Sample}

Data for these analyses came from a large, cross-sectional study designed to examine the factors associated with contraception use in women attending Family Planning Victoria. Family Planning Victoria is an independent, not-for-profit organization that is partially funded by the Victorian government. It provides clinical care in sexual and reproductive health. The Action Centres are drop-in clinics in Melbourne's central business district and Hoppers Crossing (outer metropolitan), specifically catering to people under 25 years of age. The Box Hill clinic (inner suburban) caters for all age groups with both an appointment system and drop-in services. A small administration fee is charged yearly, which gives unlimited access to the clinics where consultations are low cost or free.

Women aged 16-50 years attending Family Planning Victoria's three sites during the period April to July 2011 were recruited. These women were sexually active, with at least one male partner in the last 3 months, but were not attempting to conceive. Eligible women were invited to complete an anonymous questionnaire prior to seeing the doctor. Following receipt of the survey, participants placed their completed or uncompleted survey in a secure box. The response rate of $92 \%$ was determined by use of a numerical identifier on each survey. Eighty-five women declined to fill the questionnaire (out of 1109 approached). Eighteen surveys were excluded because of either ineligibility or incomplete questionnaires $(>50 \%$ of questions unanswered), leaving 1006 surveys for analysis.

Ethical approval was obtained from the University of Melbourne Human Ethics Advisory Group (ID 1135498) and
Family Planning Victoria Human Research Ethics Committee (S11030412000).

\section{Measures}

The questionnaire comprised 34 items and covered frequency and type of contraceptive use and potential variables affecting contraception use. The factors were classified as demographics, attitudes, and behavioral factors. The 5- to 7-minute survey is available on request to the corresponding author. ${ }^{18}$

Clients were asked three questions:

1. "In the last 3 months, have you used the following contraceptive method(s)?" Patients were asked to tick all that applied: oral contraceptive pill (OCP), male condoms, NuvaRing ${ }^{\circledR}$, diaphragm, withdrawal (pulling out), rhythm, female condom, intrauterine device (IUD), Implanon NXT®, depot medroxyprogesterone acetate (DMPA), my partner had a vasectomy, female sterilization.

2. For each contraceptive method, we asked the women to indicate how consistently the method was used: never, not usually, sometimes, most of the time, or always.

3. "In the last 3 months, have you used more than one contraceptive method at the same time? If so, which ones?" This question also helped to capture women who were using a combination of contraceptive methods.

\section{Analysis}

For the analysis, an effective contraception was defined as one that has a typical failure rate of $<10 \%$. These were the OCP, Implanon NXT ${ }^{\circledR}$, IUD/IUS, DMPA, NuvaRing ${ }^{\circledR}$, tubal ligation, and vasectomy. ${ }^{3}$ Data were entered into the statistical package MINITAB (v. 16.1.0). The statistical modeling focused on the use of condoms with an effective contraception as the major outcome of interest. Univariate logistic regression was performed with a range of explanatory variables. Backward elimination was used to remove explanatory variables with $\mathrm{p}$ value greater than 0.1 . The remaining explanatory variables were then used in an overall multivariate logistic regression model for women using condoms and an effective contraception. Statistical significance was set at 0.05 . The Hosmer-Lemeshow test, a statistical test for goodness of fit for logistic regression model was performed.

\section{Results}

Of 1006 women surveyed, 96\% reported using any contraceptive and $29 \%$ reported a combination of contraceptives used in the last 3 months. Of 1006 women, 872 women stated it was "very important" or "important" to avoid pregnancy at this stage of their life. Of these 872 women, there were 690 male condom users: 274 women used male condoms and an effective contraceptive, while 416 used male condoms only. Of note, only 67 (16\%) of the 416 solely using male condoms were consistent users. The demographics of these women are summarized in Table 1 . The study population was relatively young with a median age of 21.

Table 2 shows the percentage of male condom users (for women who stated it was important to avoid pregnancy) who 
Table 1. Demographics of Study Population $(N=690)$

\begin{tabular}{lc}
\hline Characteristic & Number of women (\%) \\
\hline Age group years) & \\
$16-19$ & $227(33)$ \\
$20-24$ & $270(39)$ \\
$25-29$ & $78(11)$ \\
$30-34$ & $36(5)$ \\
$35-39$ & $27(4)$ \\
$40-50$ & $52(8)$ \\
Highest education level completed & \\
University or postgraduate & $223(32)$ \\
Country of birth & \\
$\quad$ Australia & $516(75)$ \\
Language spoken at home & $630(91)$ \\
$\quad$ English & \\
Medical insurance & $244(35)$ \\
Health care card holder & $321(47)$ \\
Private health insurance & \\
Household income & $344(50)$ \\
$\quad$ More than \$AUS 60,000 per year &
\end{tabular}

also reported the combined use of another effective contraceptive within the last 3 months. The most popular additional effective contraception used was the oral contraceptive pill (51\%) with few women using long-acting reversible contraceptives. On univariate analysis (Table 3), those who were younger than 21 years old and born in Australia were more likely to have used an effective contraceptive with condoms compared with those solely using male condoms. Women who also used an effective contraception had greater satisfaction with their current contraception and were more likely to feel comfortable discussing contraception with their doctor but not their partner. Furthermore, these women were more likely to report that a health professional had discussed contraception with them in the last 12 months and despite reporting more than one partner in the last 3 months, these women reported a lesser likelihood of ever having an intended pregnancy, unintended pregnancy, or abortion. On multivariate analysis, the factors that remained statistically significant $(p<0.05)$ were satisfaction with current contraception, reporting a discussion with a health professional about contraception in the last 12 months, having more than one partner in the last 3 months, and having no previous

Table 2. Male Condom Users Who Also Used an Effective Contraceptive in the Last Three Months

\begin{tabular}{lc}
\hline Type of contraception & $\begin{array}{c}\text { Number of women } \\
\text { using an effective } \\
\text { contraception }(\%)\end{array}$ \\
\hline Oral contraceptive pill $^{\text {Implanon NXT }}$ & $352(51.0)$ \\
Intra-uterine device $_{\text {Depot medroxyprogesterone acetate }}$ & $77(11.2)$ \\
NuvaRing $^{\circledR}$ & $40(5.8)$ \\
Vasectomy & $14(2.0)$ \\
Tubal ligation & $8(1.2)$ \\
\hline
\end{tabular}

$n=690$ users of male condoms among women who stated it was important to avoid pregnancy. pregnancies. It was interesting to note that after adjusting for a past pregnancy, those who were currently using both male condoms and an effective contraception were more likely to have experienced an unintended pregnancy ( $p$ value is just above statistical significance, 0.054).

\section{Discussion}

In this group of women who had a strong desire to avoid pregnancy, only a minority $(40 \%)$ of condom users were also using additional effective contraception. The most common of these was the pill with very few women using a long-acting reversible contraceptive. Of concern, in the group of solely male condom users, only $19 \%$ were using condoms "with every sexual act."

There were important differences in women who used both condoms and an effective contraception compared to women who solely relied on male condoms. Firstly, dual method users were more likely to have discussed contraception with a health professional in the last 12 months. Brief counseling interventions by doctors have been shown to be effective in modifying health behaviors, especially in adolescents. ${ }^{20}$ As $85 \%$ of the population of Australia sees a general practitioner (GP) each year, GPs may be in an ideal position to improve contraceptive outcomes for individuals by using these techniques. The importance of proper education and provision of the best contraceptive for individual women was also highlighted by the finding that women using condoms and an effective contraception were less likely to report a past abortion and had greater odds of reporting satisfaction with their current contraception. There is evidence that having a positive attitude toward contraception (including condoms) was associated with an increased likelihood of use compared with nonuse. ${ }^{21-24}$ Conversely, another study has shown that those who were not satisfied with using their method had greater odds of using their method inconsistently or not at all. ${ }^{25}$ Health professionals can play an important role in increasing satisfaction of contraceptive for women by tailoring the right contraceptive for the woman and discussing and monitoring for potential side effects.

It is unclear from our questionnaire why having more than one partner may increase the likelihood of condom users being on an effective additional contraceptive. Perhaps those who reported a greater number of partners felt a greater need for advance planning in pregnancy protection whereas those with a single partner (especially if this was a regular partner) were less worried about pregnancy if it did happen. This is an area that requires further research. We also found that combination users were less likely to have experienced a past pregnancy compared to condom-only users. Although not statistically significant, it was interesting to note that when we adjusted for past pregnancy, women who used an additional effective contraceptive actually had a higher likelihood of having experienced a past unintended pregnancy.

\section{Clinical implications}

On a community level, in light of rising STIs and high unintended pregnancy rates, there is a need to broaden prevention messages to include discussing the combination of condoms and an effective contraception. Some argue for a blanket wide education that combination use "should be taught to everyone as part of sexuality and relationships 


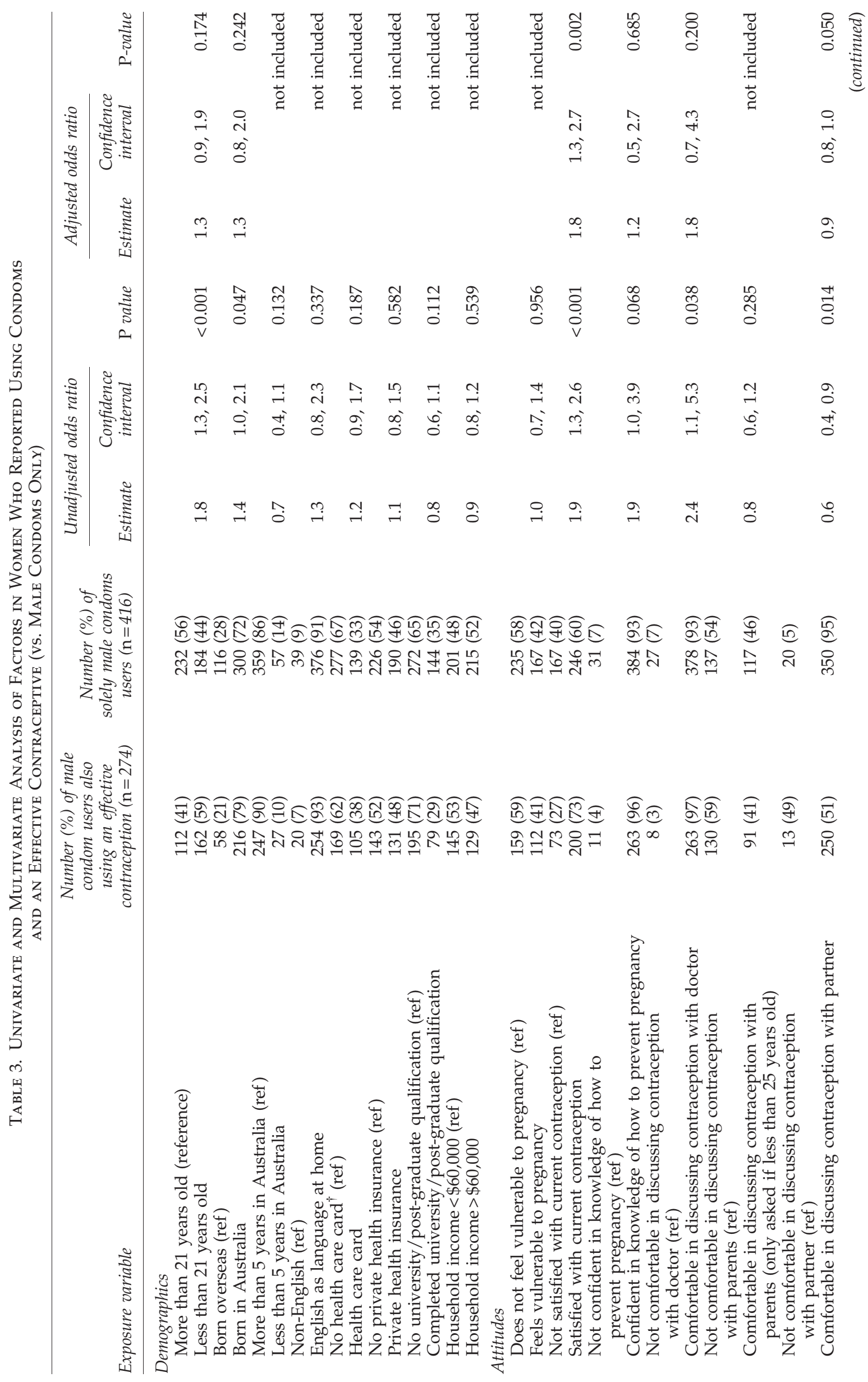




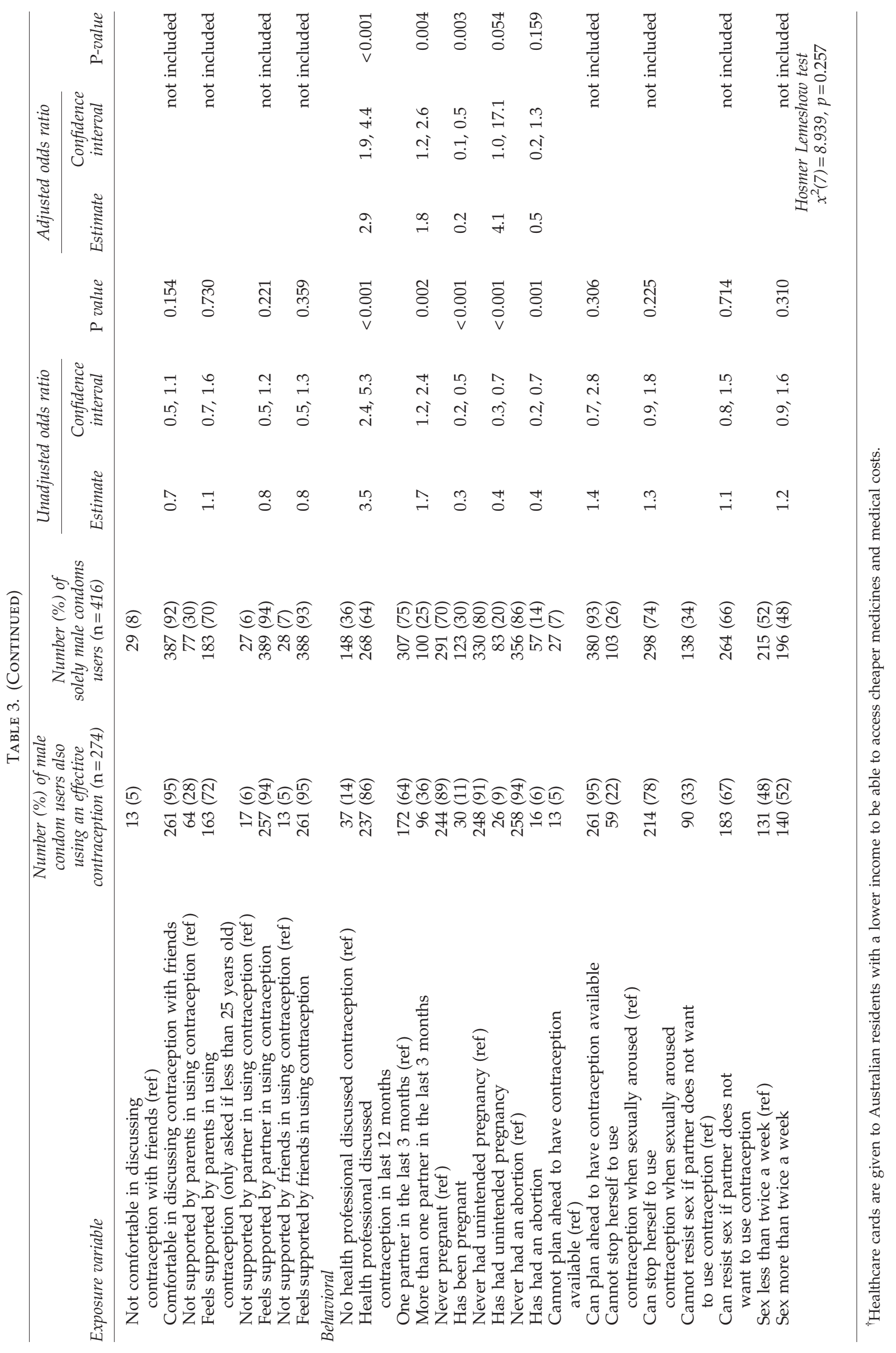


education when they are young. ${ }^{\prime 7}$ As a minimum, we must especially stress the importance of combination use for those who report new partners, have partners who have other partners, or those who have not been tested for STIs or who report multiple partners.

On the patient consultation level, every sexually active patient should have a thorough assessment of their likelihood of both exposure to STIs and unintended pregnancy. Factors that may help improve the likelihood of using both condoms and an effective contraception include ensuring a health professional regularly discusses contraception and any associated difficulties with women. If a woman has high motivation to avoid pregnancy, it may be appropriate to encourage the use of a more effective contraceptive rather than relying on the male condoms as a contraceptive. Cates agrees that the use of condoms and an effective contraception is better if there is greater concern for unintended pregnancy and the one method approach (emphasizing consistent condom use) for those with a higher likelihood of exposure to infection as defined by higher STI prevalence in the community or specific risk behaviors of the patient. ${ }^{19}$

It is important to note that the factors we found that were associated with women using male condoms only versus women also using an effective contraception were different from the factors associated with women not using an effective contraception versus those using an effective contraception published from the same study population. ${ }^{26}$ This may impact the strategies employed to increase the use of an effective contraception in women already reporting the use of male condoms on their baseline visit to a family clinic, versus women who are not using condoms at all.

\section{Limitations}

There is limited generalizability of the findings beyond the family planning population. However, the findings of this research are important as it is the first of such research in evaluating how we can encourage women not intending to conceive who are already using male condoms to also consider the addition of an effective contraception. This may help influence the practices in family planning clinics in Australia and beyond. The factors associated with women using an effective contraception with male condoms are currently unknown for nonclinical populations. The questionnaire from this study may serve as a template for future studies extended to general practice settings, hospital outpatients of obstetrics / gynecology and community settings to provide a broader understanding of how combination methods are used in other settings.

As the survey was a self-report of contraceptive use, there may be a risk of recall bias and reporting bias. Single item measures for evaluating some complex variables (attitudes of partner/parents/friends, etc.), may not be sufficiently sensitive or reliable to measure the intended variable. The survey only reported the use of contraception and not how well the methods were used. While women reported multiple methods in the last 3 months, we could not determine with certainty the temporal relationship with the use of these methods (e.g., how consistently were the methods used in combination). As the survey was a cross sectional design, the list of characteristics derived from the multivariate analysis must not be used to forecast future use of combination methods in these women. There is a need for a longitudinal study to test these characteristics as true markers for predicting women with greater odds of using condoms and an effective contraception.

\section{Further research}

As this was a subanalysis of a larger study, several suggested questions that should be included in a future specific study for combination use would be partner status (regular/ casual partner), ${ }^{27}$ whether this relationship is mutually monogamous, and why women chose to use a combination (e.g., to prevent STIs or unintended pregnancy or both, and has any doctor specifically spoken to them about using condoms and an effective contraception concurrently?) Unfortunately, the questionnaire did not distinguish women who were making their first visit to a family planning service (although the vast majority of patients attending the Family Planning Victoria service would be attending as new patients). The variable of whether a health professional had discussed contraception with the woman in the past 12 months is thus a marker for discussion of contraception outside a family planning setting (i.e., most likely the GP). It would be interesting to determine in a future study whether women were more likely to be combination users after their visit to a family planning service as opposed to a general practice.

Further qualitative research is needed to explore the reasons for using the combination of condoms and an effective contraception in Australian women, especially around the associations found in this study. Importantly, there is a glaring lack of research in the role of men in influencing the use of condoms and an effective contraception in their sexual relationship. Further research is needed to address the issues of partner dynamics and male preferences and characteristics.

\section{Conclusions}

In this group of women attending Family Planning Victoria who were not intending to be pregnant, a significant number remained at risk for unintended pregnancy. Of those who relied on male condoms alone, only $19 \%$ were using condoms with every sexual act, and only $40 \%$ of male condom users were using an effective contraceptive to prevent pregnancy. There is a need for better education and consideration of promoting effective contraception (including long-acting reversible contraceptives) in women not intending to be pregnant. This study supports the importance of ensuring a health professional regularly discusses about contraception with women and ensuring that women remain satisfied with their chosen contraceptive.

\section{Acknowledgments}

We would like to thank Family Planning Victoria staff for collecting and patients for contributing to the data presented.

\section{Disclosure Statement}

No competing financial interests exist.

\section{References}

1. Skinner SR, Hickey M. Current priorities for adolescent sexual and reproductive health in Australia. Med J Aust 2003;179:158-161. 
2. Singh S, Sedgh G, Hussain R. Unintended pregnancy: Worldwide levels, trends, and outcomes. Stud Fam Plann 2010;41:241-250.

3. Trussell J. Contraceptive failure in the United States. Contraception. 2011;83:397-404.

4. Pazol K, Kramer MR, Hogue CJ. Condoms for dual protection: patterns of use with highly effective contraceptive methods. Public Health Rep 2010;125:208-217.

5. Potter L, Oakley D, de Leon-Wong E, Canamar R. Measuring compliance among oral contraceptive users. Fam Plann Perspect 1996;28:154-158.

6. Kost K, Singh S, Vaughan B, Trussell J, Bankole A. Estimates of contraceptive failure from the 2002 National Survey of Family Growth. Contraception 2008;77:10-21.

7. Berer M. Dual protection: More needed than practised or understood. Reprod Health Matters 2006;14:162-170.

8. Godeau E, Nic Gabhainn S, Vignes C, Ross J, Boyce W, Todd J. Contraceptive use by 15-year-old students at their last sexual intercourse: Results from 24 countries. Arch Pediatr Adolesc Med 2008;162:66-73.

9. Higgins JA, Cooper AD. Dual use of condoms and contraceptives in the USA. Sex Health 2012;9:73-80.

10. Sales JM, Latham TP, Diclemente RJ, Rose E. Differences between dual-method and non-dual-method protection use in a sample of young African American women residing in the Southeastern United States. Arch Pediatr Adolesc Med 2010;164:1125-1131.

11. Sangi-Haghpeykar H, Poindexter AN, 3rd. Planned condom use among women undergoing tubal sterilization. Sex Transm Dis 1998;25:335-341.

12. Sangi-Haghpeykar H, Poindexter AN, 3rd, Bateman L. Consistency of condom use among users of injectable contraceptives. Fam Plann Perspect 1997;29:67-69, 75.

13. Cushman LF, Romero D, Kalmuss D, Davidson AR, Heartwell S, Rulin M. Condom use among women choosing longterm hormonal contraception. Fam Plann Perspect 1998;30: 240-243.

14. Darney PD, Callegari LS, Swift A, Atkinson ES, Robert AM. Condom practices of urban teens using Norplant contraceptive implants, oral contraceptives, and condoms for contraception. Am J Obstet Gynecol 1999;180:929-937.

15. Parr N, Siedlecky S. Use of "dual protection" and other combinations of contraceptive methods in Australia. Aust N Z J Public Health 2007;31:567-570.

16. Grady WR, Klepinger DH, Nelson-Wally A. Contraceptive characteristics: The perceptions and priorities of men and women. Fam Plann Perspect 199931:168-175.
17. Iuliano AD, Speizer IS, Santelli J, Kendall C. Reasons for contraceptive nonuse at first sex and unintended pregnancy. Am J Health Behav 2006;30:92-102.

18. Ong J. "Choices women make." Prevalence of and the factors associated with contraception use in women of reproductive age attending Family Planning Victoria [Masters of Medicine (by research)]. Melbourne: The University of Melbourne, 2012.

19. Cates W, Jr., Steiner MJ. Dual protection against unintended pregnancy and sexually transmitted infections: What is the best contraceptive approach? Sex Transm Dis 2002;29:168-174.

20. DiCenso A, Guyatt G, Willan A, Griffith L. Interventions to reduce unintended pregnancies among adolescents: Systematic review of randomised controlled trials. BMJ 2002;324:1426.

21. Bruckner H, Martin A, Bearman PS. Ambivalence and pregnancy: Adolescents' attitudes, contraceptive use and pregnancy. Perspect Sex Reprod Health 2004;36:248-257.

22. Roberts ST, Kennedy BL. Why are young college women not using condoms? Their perceived risk, drug use, and developmental vulnerability may provide important clues to sexual risk. Arch Psychiatr Nurs 2006;20:32-40.

23. Robinson BB, Scheltema K, Cherry T. Risky sexual behavior in low-income African American women: The impact of sexual health variables. J Sex Res 2005;42:224-237.

24. Dilorio C, Dudley WN, Soet J, Watkins J, Maibach E. A social cognitive-based model for condom use among college students. Nurs Res 2000;49:208-214.

25. Frost JJ, Darroch JE. Factors associated with contraceptive choice and inconsistent method use, United States, 2004. Perspect Sex Reprod Health 2008;40:94-104.

26. Ong J. Contraception matters: Indicators of poor usage of contraception in sexually active women attending family planning clinics in Victoria, Australia. BMC Public Health 2012;12:1108.

27. Poppen PJ, Reisen CA. Women's use of dual methods of sexual self-protection. Women Health 2000;30:53-66.

Address correspondence to: Jason Ong, MMed School of Population Health

University of Melbourne 580 Swanston Street Carlton, Victoria 3053

Australia

E-mail: J.ong@unimelb.edu.au 